\title{
Review and synthesis of a walking machine (Robot) leg mechanism
}

\author{
Tesfaye Olana Terefe ${ }^{1}$, Hirpa G. Lemu ${ }^{2, *}$, and Addisu K/Mariam ${ }^{3}$ \\ ${ }^{1}$ Mechanical Engineering Department, Mizan-Tepi University, Ethiopia \\ ${ }^{2}$ Faculty of Science and Technology, University of Stavanger, Norway \\ ${ }^{3}$ School of Mechanical Engineering, Jimma University, Ethiopia
}

\begin{abstract}
A walking machine (robot) is a type of locomotion that operates by means of legs and/or wheels on rough terrain or flat surface. The performance of legged machines is greater than wheeled or tracked walking machines on an unstructured terrain. These types of machines are used for data collections in a variety of areas such as large agricultural sector, dangerous and rescue areas for a human. The leg mechanism of a walking machine has a different joint in which a number of motors are used to actuate all degrees of freedom of the legs. In the synthesis of walking machine reported in this article, the leg mechanism is developed using integration of linkages to reduce the complexity of the design and it enables the robot to walk on a rough terrain. The dimensional synthesis is carried out analytically to develop a parametric equation and the geometry of the developed leg mechanism is modelled. The mechanism used is found effective for rough terrain areas because it is capable to walk on terrain of different amplitudes due to surface roughness and aerodynamics.
\end{abstract}

\section{Introduction}

Nowadays, the expansion of using robots in human life is becoming a more attractive area of research and development. This expansion has led many to start advancing automated machinery aimed at different applications. Over the past periods, science and technology in robotics developed and improved from fiction to reality, particularly in design and production of robots. Different applications within diverse disciplines such as computer science, medical application, fields of engineering and automation played a great role in the advancements within robotics [1]. A locomotion over a soft or hard surface by means of limbs, legs and/or wheels at low speed can be defined as a walking machine. The design and development of these machines have been widely studied by NASA for planetary exploration since the 1960s [2]. Based on the surface on which they operate, NASA developed and used three basic types of walking machines; namely wheeled, tracked and legged walking machine or robot [3-4].

Walking machines that can operate under different conditions are used for planetary, medical, engineering, agricultural task, etc. to speed up or replace human works [5-6]. The establishment of the walking machine technology started since ancient times of human civilization for conquering the machines on rough terrain surface. During the Greek

*Corresponding author: Hirpa.g.lemu@uis.no 
civilization era, for instance, mechanically powered walking machines traced from animals and humanlike appearance. In Europe, conceptual design and schematic drawing of humanlike artificial systems and mechanisms were developed [7].

Review of the historical developments of walking machines show that a wooden horse that has eight legs and jumps over high walls was constructed in 1770 [8]. The Russian mathematician, Chebyshev [9], has also designed and presented a fixed leg model for mechanical motion using clever linkage-based in the mid-1800's that moves in a straight path while the legs move up and down. Quadruped machines of a size of an elephant in General Electric Walking Truck (GEWT) was also developed in mid-1960s [9], which was a legged machine approached only by electromechanical means.

Recently, developed multi-legged robots are widely used locomotion principles in a biological system to achieve the surface mobility [10]. The legs of the robots have three degrees of freedom (DOF) which perform walking motions by adapting the body system to surface structure. In the history of the walking machine, Massachusetts Institute of Technology (MIT) contributed widely to the development of walking machines design including the hopping robot. MIT humanoid Robotics [11] group developed the concept of subsumption architecture, which is based on a strong coupling between actuators and sensors, promoting a more reactive behavior than in the classical control approaches. Based on this concept, six-legged walking machines were designed in late 1980s, which were designed with two DOF to achieve simplicity in the mechanism and low weight [12]. As a result, these robots could move on a straight line. In [13], design of a legged autonomous vehicular agent (LAVA) walking machine is reported. The legs that have swinging and lifting functions are based on the differential gear drive system. Each leg is incorporated into three DOF maximum flexibility with precis turning functions. Quite recently, in 2011, a six-legged robot, called ARMUR5, with hexagonal architecture was designed and built [14]. The robot weighs $34 \mathrm{~kg}$ and is $1.4 \mathrm{~m}$ in outer diameter. The maximum reachable velocity on flat ground is about $0.03 \mathrm{~m} / \mathrm{s}$, each leg has three DOFs, namely rotation about the vertical axis, and horizontal and vertical translations. Each DOF is actuated by DC motor using a pantographic mechanism that allows better efficiency and easier foot trajectory.

Another multi-leg walking machine is reported in [15] who designed a passively adaptive three DOF multi-legged robot with Underactuated legs. In this design, adaptive under actuation techniques with constrained design-based tools, which allow lighter and simple mobility and adapt to rough terrain, were employed. Most recently, the MicroElectromechanical System (MEMS) technology has been used in designing millimeter scale walking robots within a very improved fabrication process, called Micro-lithographic techniques. MITRE Nanosystems group designed a walking robot which involves MEMS technology as shown in Fig. 1, which has six legs having two DOF each [16].

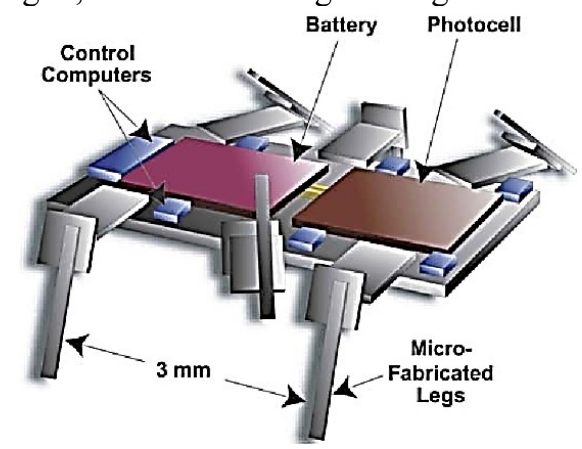

Fig. 1. High-level design of micro-robot 
Over the past periods, the robotics discipline has advanced from fiction to reality becoming the science and technique that elaborated in the design, production and use of robots $[1,17]$. According to the current researches, robotics and artificial intelligence (AI) will saturate wide sections of human's daily life by 2025 with enormous applications in a series of activities such as transport and logistics, consumer service, health precaution and home repairs [18]. In this study, the main drawbacks of existing walking machines are reviewed and kinematic and dimensional synthesis of a walking machine (robot) leg mechanism for a rough terrain is performed and the parametric equations for the proposed leg mechanism are derived.

\section{Synthesis of a leg mechanism}

In order to analyze mechanisms in early science of machine kinematics, machine elements were studied by combining the laws of their operation. In its work of "Theoretische Kinematik" of 1875, Reuleaux [19] offered many insights to the discovery of general acceptance and in recent works, he merged and extended earlier ideas, and philosophies in the study of mechanisms. His comprehensive and orderly views mark a high point in the improvement of kinematics, where he dedicated most of his work to the investigation of machine elements.

In the one hundred years that followed Reuleaux, many contributed [20] in developing the science of constructing mechanisms based on mechanics and geometry that satisfy specific motions, namely, kinematic synthesis. It was not until 1940s that numerical methods were developed [21 - 23] to design a simple but versatile mechanism known as four-bar linkage mechanism to generate the desired function using precise engineering resolutions. The input parameter functions are assigned to the input crank and the results are obtained from the output crank. Because of the nature of the linkage itself, the four-bar linkage can generate only partial tasks.

\subsection{Alternative leg mechanisms used for a walking machine}

The motion of the leg mechanism can be described in terms of position, velocity and acceleration of its all components without regard to the forces or torques that cause the motion. The components of the leg mechanism can be connected together by different types of joints that bound how the components move relative to each other. This interconnection indicates that the motion between components is constrained relative to each other. Thus, the study of the motion geometry of leg mechanisms falls under kinematics.

Different mechanisms like Klann mechanism [24] and Jansen linkages [25, 26] are widely used types of leg mechanisms for different walking machines (Fig. 2). Klann mechanism is a type of tabular mechanism to simulate the gait of leg-like animal and performance as a wheel replacement. It consists of a frame, cranks and two grounded rockers, and two couplers connected by a pivot joint, eight bar kinematic chain (Fig. 2(a)). It provides several advantages in walking machines application; step over curbs, climb stairs, travel into areas which are not accessible with wheels that don't need microprocessor control or a large amount of actuator mechanism. 

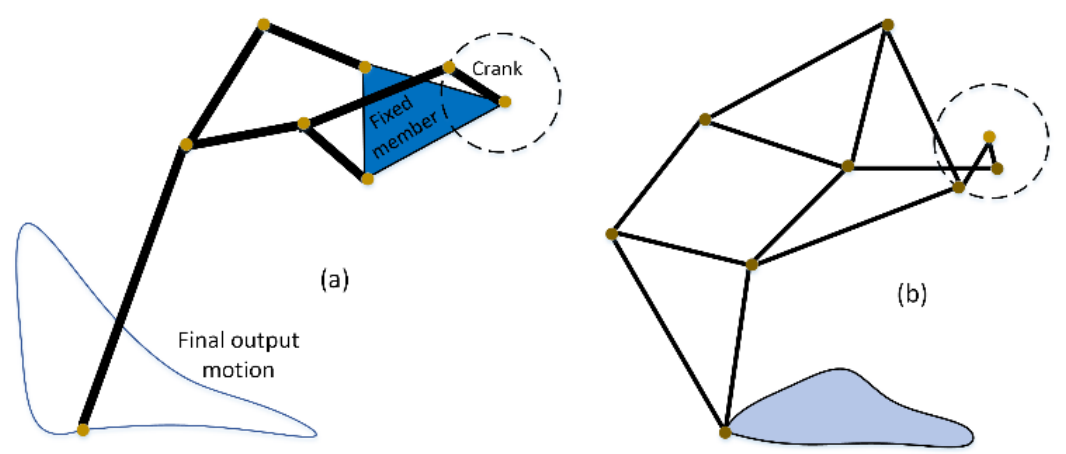

Fig. 2. Alternative leg mechanisms (a) Klann mechanism (b) Jansen linkage

Jansen's linkage mechanism is used for smooth walking motion due to its ingenious simulation of walking motion using a simple rotary input. It has one DOF planar, 11 mobile link leg mechanism that converts the rotational motion of a simple crank mechanism into a stepping motion, (Fig. 2(b)) [23]. The popularity of Jansen mechanism is due to its scalable design, efficient in energy, low payload and deterministic foot trajectory [24]. Presently, the major use of the Jansen linkage is a walking motion used in legged robotics.

\subsection{Kinematic synthesis of developed leg mechanism}

The walking mechanism can be developed by integrating a combination of linkages. In this paper, special types of four-bar mechanism that changes a point four-bar and inline-slider crank mechanism is proposed to create a mechanism that can be used in a legged walking machine to walk on a rough terrain.

As mentioned earlier, the simplest and most common closed loop linkage mechanism is the four-bar linkage (Fig. 3(a)). In this mechanism, one link is designated as a frame with four pin-joints for a single degree of freedom of the controlled motion. It has also special configurations created by making one or more links infinite in length. Slider-crank mechanism is a four-bar chain with a slider replacing an infinitely long output link, Fig. 3 (b). It is connected by three pin joints and one sliding joint.
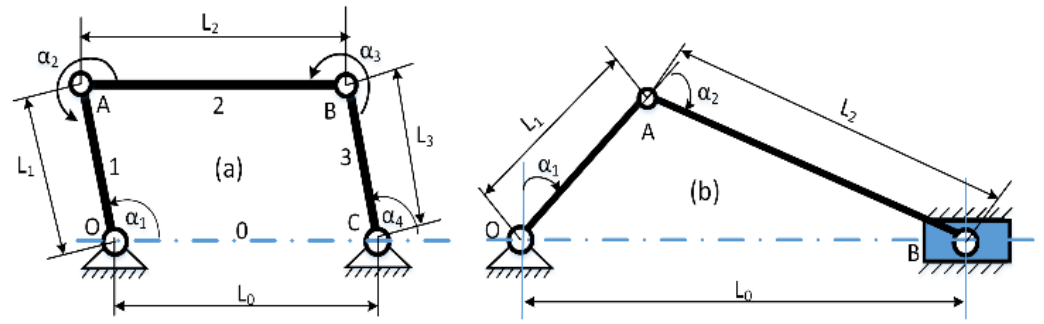

Fig. 3. Types of linkage mechanisms (a) four-bar mechanism (b) Inline slider crank mechanism

Kinematic synthesis is a process of designing a mechanism to accomplish the desired task which includes both choosing the type as well as dimensions of the mechanism. For the leg mechanism of the walking machine, the mechanism is composed of special types of four-bar change point and inline slider mechanisms in which both are integrated together to develop the required motion of back and forth, and up and down motion respectively. The change point four-bar mechanism is used for the back and forth motion of the walking machine leg. As the change point mechanism's sum of two sides is equal to the sum of the other two, the change point mechanism can be positioned such that all of the links become collinear. The parallelogram linkage given in Fig. 4(a) is a widely used type of change point mechanism. 
The frame and the coupler have the same length, and so are the two pivoting links. Thus, the four links will overlap and as a result of the collinear configuration, the motion becomes intermediate. Since the motion could stay in a parallelogram arrangement, it is referred to as a singularity configuration.
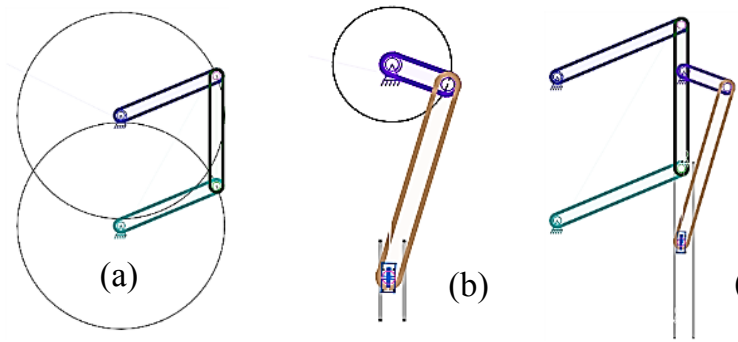

(c)

Fig. 4. Synthesis of leg mechanism

For the vertical motion, the inline slider-crank mechanism is assembled on the coupler of change point four-bar linkage, which rotates simultaneously while providing up and down motion of the leg as illustrated in Fig. 4(b) and (c). The coupler of the change point four-bar mechanism is extended beyond and can be used as a fixed frame for the slider mechanism.

The leg mechanism for up and down motion is developed from the mechanism of the inline slider-crank mechanism. This mechanism provides reciprocating linear sliding motion and it has the crank pivot coincident with the axis of this motion. The stroke is defined as the linear distance that the sliding link exhibits between the extreme positions. Because the crank and connecting arm motion is symmetric about the sliding axis, the crank angle is mandatory to perform a forward stroke similar to the return stroke. For this reason, the inline slidercrank mechanism produces balanced motion. The length of the connecting arm does not affect the stroke of an inline slider-crank mechanism. However, a shorter connecting arm yields greater acceleration values.

The gear assembly is used to transfer motion between links from single central input rotary motion. The arrangements of the gears are designed to provide input motion for the change point four-bar mechanism and the inline slider-crank mechanism. The coupler of the change point four-bar mechanism is used as a fixed frame for the inline slider-crank mechanism having a prismatic joint at its one end which helps the connecting arm of the slider to slide in to develop the up and down motion of the leg.

\section{Dimensional synthesis of leg mechanism}

Dimensional synthesis deals with the determination of kinematic (link lengths, offsets, etc.) of the leg mechanism to satisfy the required motion characteristics. For the developed leg mechanism, a dimensional analysis of both change point four-bar and inline slider-crank mechanisms are formulated analytically using parametric equations.

With this mechanism, the drive crank and follower crank have the same length as well as that the connecting rod or coupler and the ground pivots have the same length. The drive crank and the follower crank will always have the same angular velocity. The dimensional synthesis of the change point four-bar evaluates the perfect structure that can provide the maximum workspace. The dimensional synthesis is carried out by controlling the following geometrical parameters such as the link length, and the angle between the link as given in Fig. 5. The typical structures with the dimensions are drawn and, the angle and the displacements are identified. The relations between the linkage elements for the four-bar synthesis in Fig. 5(a) are given in Eqs. (1) - (3). 

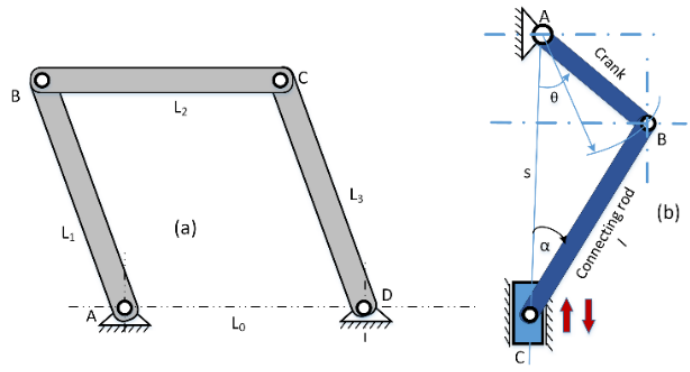

Fig. 5. Mechanism dimensional synthesis (a) four-bar linkage (b) crank

$$
\begin{gathered}
L_{0}=L_{1}=L_{2}=L_{3} \\
A B / / C D, B C / / A D \\
\overline{A B}+\overline{B C}=\overline{A D}+\overline{D C}
\end{gathered}
$$

One or more data of the frequency of oscillation, the speed at different positions and range of motion of the slider may be provided. Then, the required task will be finding the dimensions of the members of the leg mechanism and position of the slider such as to have the desired motion of the leg mechanism. The relation between the link lengths of the crank mechanism are determined from Fig. 5(b) parametrically to formulate the general equation of its dimensions as given in Eqs. (4) to (7).

$$
\begin{gathered}
s=l \cos \alpha+r \cos \theta \\
r \sin \theta=l \sin \alpha
\end{gathered}
$$

Applying Cosine law (referring to Fig. 5(b)) gives

$$
l^{2}=r^{2}+s^{2}-2 r \cos (\theta)
$$

Upon using trigonometric identities and simplifications, the displacement $s$ is expressed as a function of the geometric parameters $r, l$ and $\theta$ as

$$
s=r \cos \theta+\sqrt{l^{2}-r^{2} \sin ^{2} \theta}
$$

\section{Discussion of results}

The rocker-bogie suspension for the wheeled system and legged walking machine were among the walking robot systems that were introduced and used by NASA for the planetary application. The main drawbacks of the wheeled suspension system are that they need flat surface and their mobility performance over rough terrain is very less compared to the legged mechanism. Even those designed for rough terrain cannot pass over obstacles or holes greater than their wheel diameter.

In addition, due to track system damages, i.e. the contact surface over which they operate, it is infeasible to make the wheeled system applicable on rough terrain. In comparison, the legged mechanism is the most suitable design for rough surface. Due to this reason, this study aims to explore the working principle and advance the design or synthesis of a leg mechanism of walking machine (robot). From recent developments, it is possible to witness that legged walking machines use much complex mechanisms. The developments in the drive system, i.e. small motors with high drive power, has enabled to easily actuate each DOF using independent motors. 
To study the performance of the designed mechanism, the mechanism geometry is used to calculate the position, velocity and acceleration of points on different joints of the mechanisms and their motion trajectories are traced (Fig. 6). Furthermore, motion analysis in SolidWorks was conducted in order to be able to predict the response of the motion of the walking machine leg for a given external input parameter.

The Denavit-Hartenberg (DH) convention, which is a commonly used approach for selecting frames of reference in robot applications, is employed to enable construction of the direct kinematics function by decomposition of the individual transformations into a homogeneous transformation matrices that are coded in MATLAB. The generated motion path (Fig. 6 (right)) shows the typical single leg motion path of the walking machine.

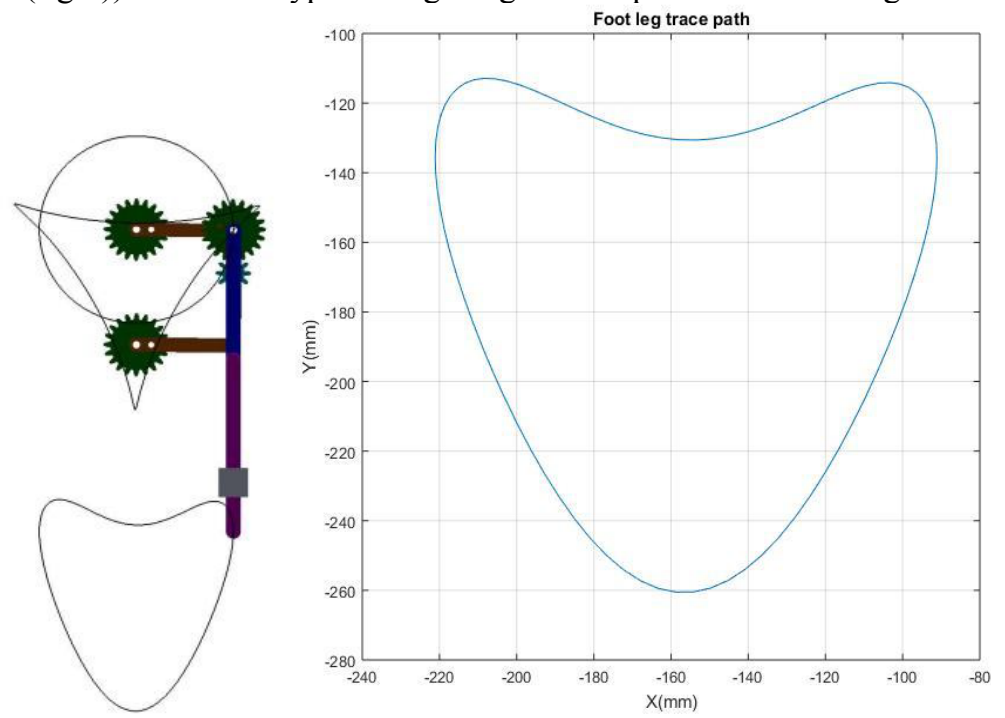

Fig. 6. Generated motion path for a single leg mechanism

The fact that the leg can move forward and backward as well as up and down demonstrates its ability to walk on a rough terrain. Based on this, the height of the obstacle or the amplitude of the rough terrain over which the walking machine can come over can be identified. Based on the assumed boundary conditions, the result shows that the walking machine can climb over a rough terrain having up to $150 \mathrm{~mm}$ height and horizontal distance of $130 \mathrm{~mm}$.

The response of a single leg shows that the motion has a smooth profile with no external input function. Direct relationship between the motion parameters, i.e., displacement, velocity and acceleration is observed and all of them are time dependent. To generate the motion path, the design considered a single motor drive and the linkages is arranged in a manner that it can transfer motion between links. This reduction of the number of motors reduces the weight of the machine and results in less power consumption to derive the leg mechanism. Another issue addressed in the design is the encountered stability problem and its complexity to design for walking machines. This issue is simplified in the design of the arrangement of the movement of legs by allowing the walking machine to touch the ground with three legs at a time. This keeps the stability while it is in a motion. The suspension system of the mechanism will be designed using a combination of linkages, which still requires further detailed analysis. 


\section{Conclusions}

This paper presents part of a work done on design and development of a walking machine and analysis of the kinematic performance when used on rough terrain. Based on review of previous designs and reported publications, the part reported in this paper focuses on synthesis of a new mechanism that can walk on rough terrain. The motion mechanism is designed, modelled and the motion parameters are coded in MATLAB. As part of this work, the following can be highlighted as the withdrawn conclusions:

- Special types of four-bar mechanisms are used to enable effective motion of the walking machine leg mechanism for walking operation.

- Simple linkage integration is proposed in the synthesis to generate motion path for the leg mechanism of a walking machine.

- Parametric equations based on mechanism geometry are developed and formulated in homogenous matrices. This helps to make the design scalable to any size for constructing the machine.

- The number of actuator is decreased and this reduces the energy consumption.

The support, comments and suggestions of Mr. Tadele B. Tuli Mr. Hassen Nigatu and Mr. Johnson Santhosh are deeply appreciated.

\section{Reference}

1. I. Zamalloa, R. Kojcev, A. Hernández, I. Muguruza, L. Usategui, A. Bilbao, V. Mayoral, Cornell University, arXiv:1704.08617 (2017).

2. R. Knight, U. Nehmzow, Technical Report, Colchester University of Essex, Contract No.: CSM375 (2002).

3. V. S. Bartsch, Künstl Intell., 28, $127-131$ (2014).

4. G. Pratt, IEEE Rob. Autom. Mag., 7, 15 - 19 (2000).

5. S. M. Pedersen, H. Fountas, H. Have, B. S. Blackmore, Precis. Agric., 5, 589-596 (2004).

6. N. Yadav, B. Bhardwaj, S. Bhardwaj, IOSR-J. Mech. Civil Eng., 12, 64-67 (2015).

7. K. Nonami, R. K. Barai, A. Irawan, M. R. Daud, In: Hydraulically powered hexapod robots: Design, Implementation and Control, 19-40 (2014).

8. D. Giesbrecht, Master Thesis, University of Manitoba, Canada, (2010).

9. S. Kim, P. M. Wensing, Found.Trends Rob. 5, 117-190 (2017).

10. Y. F. H. Kimura, A. H. Cohn, Int. J. Rob. Res., 22, 187-202 (2003).

11. D. Spenneberg, F. Kirchner, In: Climbing \& Walking Robots, Towards New Applications, eds M. Xie, S. Dubowsky, et al., 197-218 (2007).

12. T. Murali, S. Perumal, R. Mohan, P. Palanisamy, Imperial J. Interdiscip. Res., 2, 476-483 (2016).

13. T. Zielinska, J. Heng, Mechatron., 12, 737-754 (2002).

14. Q. Bombled, Doctoral Dissertation. Université de Mons, Belgium (2011).

15. O. Y. Rojas, N. Kanner, A. M. Dollar, ASME Int. Des. Eng. Technical Conf. \& Comput. Inf. Eng., 5A, 1 - 10, Boston, USA (2015).

16. D. Ellenbogen, J. C. Routenberg, MITRE Nanosystems Group (2000).

17. O. Kazuya, H. Toshihiro, F. Masakazu, S. Nobuhiro, S. Mitsuharu. IHI Eng., 42,103-107 (2009).

18. A. C. Staugaard Jr., NJ, Englewood Cliffs: Prentice-Hall (1987).

19. T. Koetsier, In: Ceccarelli M. (eds) Int. Symp. Hist. Mach. Mech. Proceed. HMM 2000. Springer, Dordrecht (2000).

20. F. Freudenstein, Reson. 15, $740-767$, (2010).

21. S. Bai, Mech. Mach. Theory, 118, 53-64 (2017).

22. G. Wu, S. Bai, Rob. Comput. Integr. Manuf., 56, 55 - 65 (2019).

23. J. Zhang, H.-w. Guo, J. Wu, G.-J. Gao, A. Eriksson, Acta Astronaut. 151, 420-431, (2018).

24. N.G. Lokhande and V.B. Emche, Int. J. Mechan. Eng. Comput. Appl. 1, 13-16, (2013)

25. S. Nansai, M. R. Elara, M. Iwase, Procedia Eng., 64, 1562-1571 (2013).

26. S. Nansai, N. Rojas, M.R. Elara, R. Sosa, M. Iwase, Adv. Mech. Eng., 7, 1-18 (2015). 